\title{
First observation of production of three massive gauge bosons
}

\author{
Philip Chang ${ }^{a, b, *}$ \\ ${ }^{a}$ University of California San Diego, \\ San Diego, USA \\ ${ }^{b}$ On behalf of CMS Collaboration \\ E-mail: philip@ucsd.edu
}

\begin{abstract}
We present the search for heavy triboson production, specifically targeting the production of $W W W, W W Z, W Z Z$ and $Z Z Z$ processes in multileptonic final states with $137 \mathrm{fb}^{-1}$ of data collected by the CMS detector during Run 2 of the LHC at $\sqrt{s}=13 \mathrm{TeV}$. An event selection consisting of identically charged dileptons and trileptons is constructed to primarily study the $W W W$ process, while the $W W Z, W Z Z$ and $Z Z Z$ processes are explored in four, five and six lepton final states. The analysis utilizes both traditional cut-based and multivariate techniques using boosted decision trees.
\end{abstract}

40th International Conference on High Energy physics - ICHEP2020

July 28 - August 6, 2020

Prague, Czech Republic (virtual meeting)

\footnotetext{
${ }^{*}$ Speaker
} 


\section{Introduction}

The discovery of a new particle that is consistent with the Standard Model (SM) Higgs boson advanced our knowledge of origin of mass and the electroweak symmetry breaking in a major way [1, 2]. However, there are still many questions that remain in the electroweak sector [3]. Following are some of the questions that remain in the electroweak sector:

1. Is the newly found Higgs boson the SM Higgs boson?

2. Is it the only scalar particle in nature? Or are there more states involved in electroweak symmetry breaking?

3. Are all multi-boson interactions SM-like?

Studying the multi-boson final states at the Large Hadron Collider (LHC) at CERN can help answer these questions. In particular, the triboson production processes contain many multi-boson interactions and studying the process can help understand the electroweak sector better. There are four triboson $(V V V)$ production modes: $W W W, W W Z, W Z Z$, and $Z Z Z$ modes. Figure 1 shows some of the Feynman diagrams that contribute to the process. In addition to the three massive gauge bosons radiating off of the virtual quark propagator, there are diagrams with triple-gauge-boson couplings, quartic-gauge-boson couplings, and Higgs-gauge-boson couplings involved at leading order.
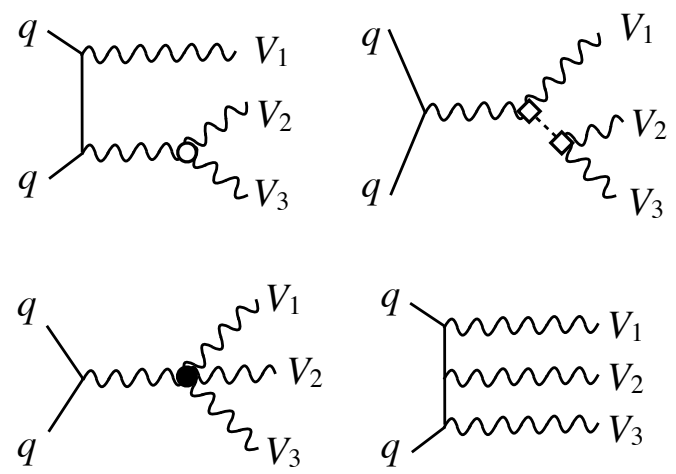

Figure 1: Tree-level Feynman diagrams of three boson productions $\left(V_{i}=W, Z\right)$, where the triple gaugeboson coupling, quartic gauge-boson coupling, and Higgs-gauge-boson couplings are marked by $\circ, \bullet$, and $\diamond$, respectively.

There were no analyses for the search for triboson production of three massive bosons before the LHC experiments. Only a handful of measurements have been carried out for the search for a triboson production at the LHC. The ATLAS experiment has published an analysis on the search for the $W W W$ production with the $8 \mathrm{TeV}$ Run 1 data set [4] and a search for the $W V V$ with the $13 \mathrm{TeV}$ data set [5]. The latter search showed evidence for $W V V$. The CMS experiment has also published an analysis on the search for the $W W W$ production with the $13 \mathrm{TeV} 2016$ data set [6].

Here, we present the search for the $W W W, W W Z, W Z Z$, and $Z Z Z$ productions in same-sign (SS) dilepton, three, four, five, and six-lepton final states. Previously only up to the four lepton 
final states have been explored in [4-6]. We present the first search for the production of $W Z Z$ and $Z Z Z$ in five and six lepton final states, respectively. Also the search for the combined production of three massive gauge boson $V V V$ is presented as well.

\section{Search strategy for $p p \rightarrow V V V$}

The $W W W$ production cross section is the highest among the three massive gauge boson productions and decreases as each $W$ is swapped with a $Z$. The total cross section ranges from $0.51 \mathrm{pb}$ to $0.04 \mathrm{pb}$. The portion of events that fall in one of the five categories of lepton final states are very rare. With the full Run 2 data set of CMS Collaboration, the total number of leptonic final states of three massive gauge boson processes are between 10s to 1000 s events depending on the process. This highlights the fact that the process was not accessible until the Run 2 data set.

The main feature of the processes is the multi-lepton signature. Therefore, it is pertinent that the experiment reconstructs the leptons very well. In fact, electrons and muons are some of the best reconstructed and measured particles at CMS Collaboration by combining tracker, calorimeter, and muon chambers measurements. The resolution of the electrons and muons in the momentum range of interest is $1-2 \%[7,8]$.

\begin{tabular}{|c|c|c|c|c|c|}
\hline & Same-sign & 3 leptons & 4 leptons & 5 leptons & 6 leptons \\
\hline  & $\begin{array}{l}W_{ \pm} \rightarrow I^{ \pm} V \\
W^{ \pm} \rightarrow I^{ \pm} V \\
W^{+} \rightarrow q q\end{array}$ & $\begin{array}{l}W \rightarrow I V \\
W \rightarrow I V \\
W \rightarrow I V\end{array}$ & $\begin{array}{c}W \rightarrow I V \\
W \rightarrow I V \\
Z \rightarrow I I\end{array}$ & $\begin{array}{l}W \rightarrow I V \\
Z \rightarrow I I \\
Z \rightarrow I I\end{array}$ & $\begin{array}{l}Z \rightarrow I I \\
Z \rightarrow I I \\
Z \rightarrow I I\end{array}$ \\
\hline 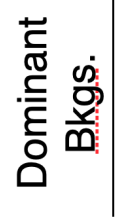 & 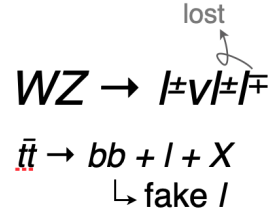 & $\left|\begin{array}{r}W Z \rightarrow I V I I \\
\bar{t} \rightarrow b b+\| l+X \\
L \text { fake } I\end{array}\right|$ & $\begin{array}{c}Z Z \rightarrow I I I I \\
\mathrm{ItZ} \rightarrow \mathrm{IIII}+\mathrm{bbX}\end{array}$ & $\begin{array}{c}Z Z \rightarrow \text { IIII } \\
+ \text { fake lep }\end{array}$ & $\begin{array}{c}Z Z \rightarrow \text { IIII } \\
+2 \text { fake lep }\end{array}$ \\
\hline
\end{tabular}

Table 1: The targeted signal in each lepton channel and the dominant backgrounds in the channel is listed.

As mentioned in the previous section, five categories of leptonic final states are studied: SS, three, four, five and six-lepton final states. By separating events into different lepton final states, different three massive gauge boson processes can be targeted strategically. The Table 1 shows the targeted signal production modes for each lepton final states with dominant background processes identified. Generally the background processes can be split into two types. The first is diboson backgrounds and the second is associated production of massive gauge bosons and top quarks. The first can be reduced through lepton identification and flavor choices, and the second can be reduced through $b$ tagging algorithms.

Several kinematic variables are used to suppress the background where the details of the full selection can be found in [9]. To further improve the sensitivity of the analysis to the signal, boosted decision tree technique is used for the SS, three, four-lepton channels. Dedicated BDTs were trained targeting two different sets of background processes for each channel. Then either by selecting a 
high signal-to-background ratio bin, or by binning the two-dimensional phase-space from two BDT output scores, the signal sensitivity was improved by 5\%-15\% depending on the channel.

The dominant backgrounds are estimated using data-driven estimate methods. For backgrounds from the diboson process and associated production of massive gauge bosons and top quarks, control regions are devised extrapolated to the signal regions based on monte-carlo simulation with relevant uncertainties. For backgrounds with mis-identified leptons, the so-called fake-rate method is used to estimate the background. The relevant uncertainties in each lepton categories vary depending on the channel but are all smaller than the data statistical uncertainty.

In summary, there are a total of 21 bins in the final fit. For SS channel, the three categories of events based on the flavor of the leptons, $e e, e \mu, \mu \mu$ are convoluted with the three categories based on the jets, one jet, two jets with dijet mass on- $W$ boson mass, and off- $W$ boson mass, that adds up to total of 9 bins. For three-lepton channel, there are three categories based on the number of same-flavor-opposite-sign dilepton pair (SFOS) in the event. For four-lepton channel, there are a total of 7 bins with events binned based on BDT scores. Lastly, for five and six-lepton channel, there are one bin each, as the statistics of the events are low.

\section{Results}

For signal extraction two fitting hypotheses are tested: one with contributions from feynman diagrams with Higgs boson included as part of the signal and another with vice versa. Also, for each hypothesis, the contributions for individual $V V V$ production modes were floated together with a single signal strength parameter, or individually with four different signal strength parameters. Therefore there are a total of four fitting configurations explored.

The final post-fit yields for Higgs boson production processes taken as part of signal with individual production modes floated are shown in Figure 2. The yields for the 21 bins are shown. The bins are organized by channels but generally the expected significances are higher for bins on the right. The pull plot shows that the agreement between signal plus background hypothesis compared to the data shows good agreement.

From these fit configurations, the sensitivities for each individual mode as well as the combined production of three massive gauge boson with Higgs boson production processes contribution taken as a part of the signal are obtained. The significances are found to be, $5.9 \sigma(5.7 \sigma)$ for combined production of three massive gauge bosons, and $3.3 \sigma(3.1 \sigma)$ for $W W W, 3.4 \sigma(4.1 \sigma)$ for $W W Z, 1.7 \sigma$ $(0.7 \sigma)$ for $W Z Z$, and $0.0 \sigma(0.9 \sigma)$ for $Z Z Z$. Therefore this results marks the first ever observation of production of three massive gauge boson in proton-proton collisions, and also provides individual evidences for $W W W$ and $W W Z$. The fitted signal strengths are also shown in Figure 3. They show good agreement with the Standard Model expectations within the uncertainty of the measurement and no direct sign of significant deviations have been observed.

\section{Summary}

In summary, proton-proton collision data at $\sqrt{s}=13 \mathrm{TeV}$ recorded with the CMS experiment and amounting to $137 \mathrm{fb}^{-1}$ were used to observe the production of three massive gauge bosons. The significance of the observation is $5.7 \sigma$ with $5.9 \sigma$ expected. For $W W W(W W Z$ ) production, 


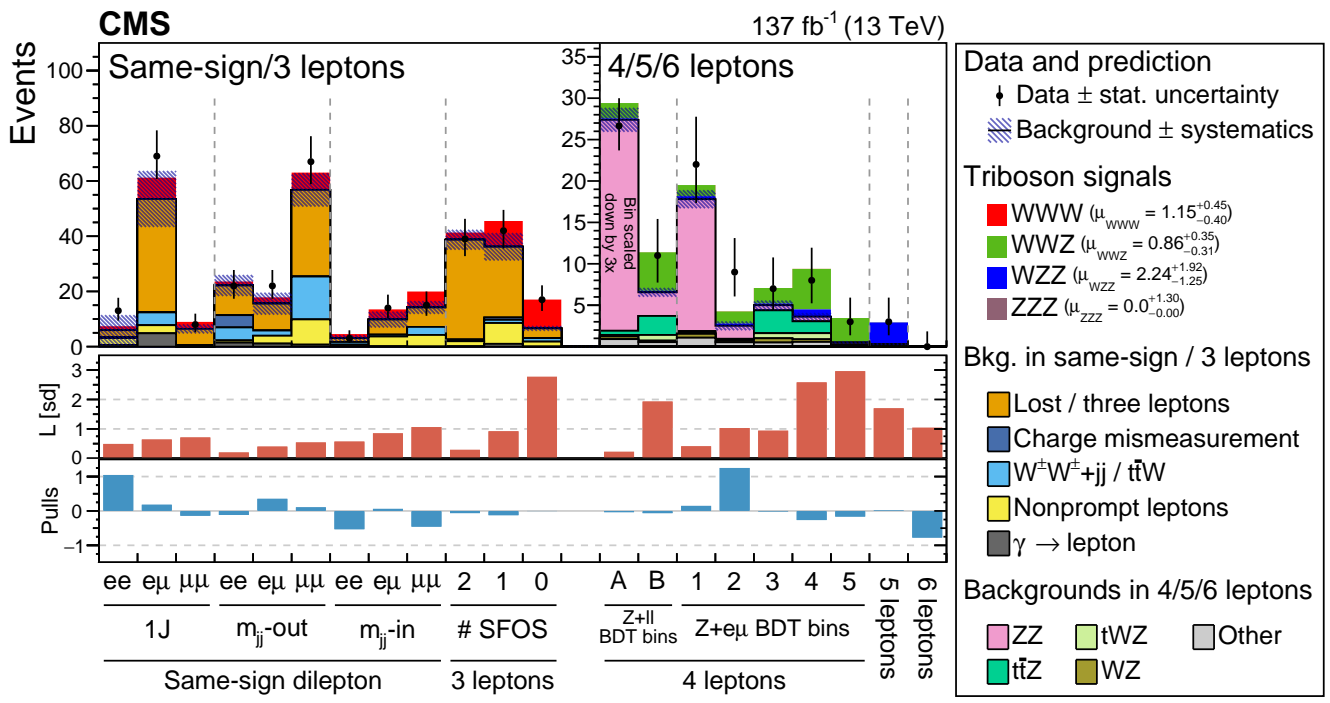

Figure 2: Comparison of the observed numbers of events to the predicted yields after fitting. For the $W W W$ and $W W Z$ channels, the results from the BDT-based selections are used. The $V V V$ signal is shown stacked on top of the total background. The points represent the data and the error bars show the statistical uncertainties. The expected significance $L$ in the middle panel represents the number of standard deviations (s.d.) with which the null hypothesis (no signal) is rejected; it is calculated for the fit for combined production of $V V V$. The lower panel shows the pulls for the fit result.

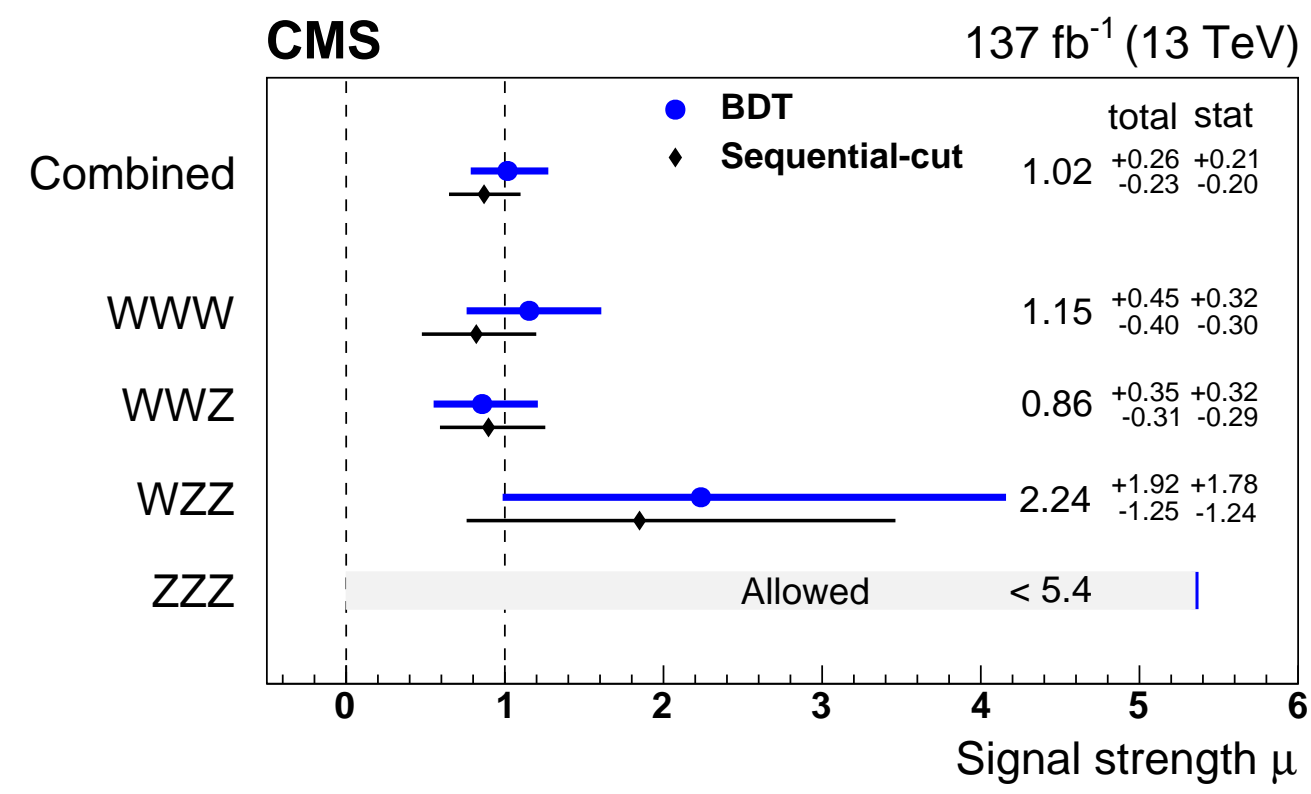

Figure 3: Best fit values of the signal strengths for the BDT-based analyses (blue solid circles) and the sequential-cut analyses (black solid diamonds). The error bars represent the total uncertainty. For ZZZ production, a 95\% confidence level upper limit is shown. The stated numerical values correspond to the BDT-based analysis.

the observed significance is $3.3 \sigma(3.4 \sigma)$ compatible with $3.1 \sigma(4.1 \sigma)$ expected. Measured cross 
sections for $W W W, W W Z$, and $W Z Z$ production and an upper limit for $Z Z Z$ production are in agreement with the expectations of the standard model. This proceeding reports the evidence for $W W W$ and $W W Z$ production and the first observation of the combined production of three massive gauge bosons.

\section{References}

[1] CMS Collaboration, Phys. Lett. B 716 (2012), 30-61

[2] ATLAS Collaboration, Phys. Lett. B 716 (2012), 1-29

[3] C. Quigg, Ann. Rev. Nucl. Part. Sci. 65 (2015), 25-42

[4] ATLAS Collaboration, Eur. Phys. J. C 77 (2017) no.3, 141

[5] ATLAS Collaboration, Phys. Lett. B 798 (2019), 134913

[6] CMS Collaboration, Phys. Rev. D 100 (2019) no.1, 012004

[7] CMS Collaboration, CMS-DP-2020-037, https://cds.cern.ch/record/2725004.

[8] CMS Collaboration, JINST 13 (2018) no.06, P06015

[9] CMS Collaboration, Phys. Rev. Lett. 125 (2020) no.15, 151802 